\title{
Workshop Proceedings
}

\section{A Strategic Vision for Telemedicine and Medical Informatics in Space Flight}

\author{
DAVID R. WILLIAMS, M.D., ${ }^{1}$ RASHID L. BASHSHUR, Ph.D., ${ }^{2}$ SAM L. POOL, M.D., ${ }^{1}$ \\ CHARLES R. DOARN, M.B.A., ${ }^{3}$ RONALD C. MERRELL, M.D., ${ }^{3}$ \\ and JAMES S. LOGAN, M.D. ${ }^{1}$
}

\section{INTRODUCTION}

$\mathbf{T}$ HIS SUMMARY REPORT describes the organization, deliberations, and recommendations of a Strategic Planning Workshop convened by the National Aeronautics and Space Administration (NASA) on November 9-10 in Houston, Texas. Earlier, NASA developed a strategic plan outlining telemedicine and medical informatics initiatives. ${ }^{1}$ This plan, however, did not contain specific instrumentalities in sufficient detail to achieve precise goals in direct support of human space flight. The purpose of this Workshop was to provide further direction for the development of telemedicine and medical informatics in providing medical support in this endeavor. The Workshop derived from the recognition that: (1) telemedicine and, more generally, medical informatics are evolving into important and effective modalities for the rapid delivery of medical care and the development of interactive medical care systems for remote locations; and, (2) NASA must position itself to capitalize on successful terrestrial application of telemedicine and medical informatics to facilitate their integration into the development and provision of space medicine.
This report is organized into five sections. The first includes an overview and background providing: (1) a brief discussion of NASA's early participation in telemedicine as well as the current use of remote medical assessment; (2) discussion of the role of telemedicine and medical informatics related to human space flight; and, (3) the Workshop organization and charges made to participants (a list of participants is provided in Appendix A). The remainder consists of four sections. The first identifies and summarizes vision statements by each of the working groups in the Workshop pertaining to telemedicine and medical informatics-based medical support systems for human space flight. The second section focuses on requirements deemed necessary for the achievement of goals delineated in the vision statement. The third section includes specific recommendations for NASA. And, the last section provides a summary of the high priority recommendations and concluding remarks.

\section{OVERVIEW AND BACKGROUND}

Telemedicine and medical informatics serve as significant tools for management, support,

\footnotetext{
${ }^{1}$ Space Life Sciences Directorate, NASA Johnson Space Center, Houston, Texas.

2University of Michigan Telemedicine Center, Ann Arbor, Michigan.

${ }^{3}$ Medical Informatics and Technology Applications Consortium, Virginia Commonwealth University, Richmond, Virginia.
} 
and delivery of health care to as well as monitoring the well being of humans in the extreme environment of space exploration. ${ }^{2}$ Responsibility for astronaut medical care, including telemedicine for human space flight, lies with the NASA Johnson Space Center (JSC). To meet the growing challenge of effectively integrating medical informatics and telemedicine into space medicine, the NASA JSC organized this Workshop on Telemedicine and Medical Informatics with a specific focus on medical support for human space flight.

NASA's long-standing interest in telemedicine has been integral to its mission. The first American astronauts in space, beginning in 1961 with Project Mercury, were monitored from ground stations via telemetry using one of the world's first miniaturized electrocardiographs in association with the NASA communications network. As early terrestrial experiments using television technology were developed and designed to bring medical care to patients in remote locations, NASA's telemedicine program was developed to support human space flight. Its early efforts were focused on integrating evolving medical and communications technologies and assessing their applicability to space flight. These capabilities were verified in a remote medical setting on an Indian reservation. The STARPAHC project, a cooperative program between NASA, the Indian Health Service, and the Papago (now Tohono O'dham) Indian Tribe in Arizona, was designed to enhance access to health care on the reservation. ${ }^{3-5}$ In 1989, NASA supported the use of space-based communication facilities to facilitate international telemedicine in the Telemedicine Spacebridge to Armenia after the earthquake there. The operational strengths and limitations of interactive video of this effort were also identified. ${ }^{6}$ More recently, some of the clinical constraints and cost inefficiencies of videoconferencing have prompted NASA to support the development of broadband Internet-based telemedicine. ${ }^{7}$

NASA's efforts to develop a telemedicine capability for space missions have included the provision of a flight qualified advanced cardiac life support (ACLS) system that was flown on the STS 90 Neurolab Mission. To gain more experience in the integration of medical and com- munications technologies, NASA developed the Telemedicine Instrumentation Pack (TIP) to connect with the Space Shuttle and the ISS KuBand downlink communications capability. ${ }^{8}$ The TIP is a computer-based system that can relay video imagery of the eye, ear, nose, throat, and skin. It is also equipped with several medical devices for monitoring oxygen saturation in the blood, blood pressure, and heart rate, acquisition of an electrocardiogram, and an electronic stethoscope. The TIP was successfully evaluated during a space shuttle flight in 1998. Although now considered obsolete, the TIP was an important first step in developing medical support for eventual exploration class missions.

Although telemedicine has been an integral part of NASA space flight operations since the beginning of human space flight, it has not matured as rapidly as terrestrial telemedicine. This is principally due to two factors: (1) the astronaut population is a relatively healthy population; and (2) the absence of any life-threatening medical problems during space flight. However, the joint US/Russian Shuttle/Mir experience led to serious concerns regarding physiological, psychological, and medical care aspects of long duration missions. Environmental contingencies including fire, loss of pressure, and contaminants in the air exacerbate these concerns. Indeed, the microgravity environment significantly complicates the traditional context of care including prevention, diagnosis, and treatment. Further complicating the situation are the actual distances and isolation of space travel, which render them incomparable to terrestrial situations. For these reasons, most earth-based medical care delivery models are not readily applicable in outer space. At the same time, telemedicine has proven to be an effective tool in addressing medical issues on board the Mir space station.

These operational realities require the development of flight-certified clinical equipment, procedures, and onboard personnel that can function with increasing levels of autonomy, especially in the absence of real-time communications. When communications (perhaps delayed) are available between onboard personnel and ground-based medical expertise, the multilateral nature of long duration and expe- 
dition class missions will require links to a broad range of clinical expertise located in major health centers within the United States and in other countries.

\section{METHODS}

This Workshop was organized by NASA to support and facilitate the strategic initiatives for integrating telemedicine and medical informatics into operational space medicine. Participants represented a broad spectrum of expertise in telemedicine, medical informatics, and space flight, both internal and external to the agency. Prior to the creation of work groups, NASA medical personnel and its external advisors provided participants with extensive information regarding current telemedicine-related activities.

Workshop participants were divided into three working groups. Each group was asked to address four tasks. (1) Develop a vision statement for NASA to consider in the development of telemedicine in support of human space flight. (2) Develop a set of requirements necessary to support the long-term needs of NASA in the area of telemedicine. (3) Outline specific approaches or methodologies that might be employed to address both the vision statements and requirements. (4) Offer recommendations pertinent to the question: "What does NASA need to develop with regard to telemedicine for long space flight?" Subsequently, the groups reported their deliberations, findings and recommendations in plenary sessions.

\section{RESULTS}

\section{Vision statement}

The vision statements developed by the working groups reflected a serious concern for providing high-quality health care for astronauts under all conditions of space flight through the integration of medical information technologies and collaboration among supporting entities. The groups concurred that NASA should acquire, develop, test, validate, and implement information management and telemedicine applications to support an evi- dence-based approach to medical practice in space aimed at minimizing the negative impacts of microgravity and potential time/distance constraints. The consensus of the groups was to encourage NASA to forecast various scenarios of medical, environmental, and occupational risks for long-duration space flight, and to develop specific responses to these risks including the requirements and resources necessary to address them. More specifically, explicit protocols, onboard capabilities, and external medical support linkages should be developed to address each scenario. Moreover, a rigorous system of ongoing quality improvement would maximize mission success.

The following are the specific vision statements for each of the three groups.

Group 1: Astronauts should be provided the highest quality health care available through the integration of medical and information technologies that develop equipment and skills sufficient to assure mission success and to maximize its impact, and assure the safety of astronauts. The medical and health care, at a minimum, must meet the highest standards of evidence-based care available on earth.

Group 2: The mission crew (both on board and ground-based) must be educated and empowered to achieve clinically acceptable outcomes through the use of advanced technology and a medical care delivery model designed specifically for space flight. The guiding principle in this regard must be maximizing the probability of mission success.

Group 3: Requirements (needs) for telemedicine in prolonged space flights must be determined first. Subsequently, the appropriate technology and training must be developed and implemented. To the extent possible, the communication technology and level of medical practice must meet or exceed terrestrial standards of care.

\section{Requirements}

The second task for the working groups was to explore and identify a set of requirements necessary for developing a health and medical support system to support long-duration space flight. Although there was some discussion regarding the difficulty of establishing meaning- 
ful and highly specific requirements in the absence of a clearly defined mission scenario, the groups were able to establish a general basis for requirements' development.

It was generally agreed NASA does and will continue to provide high quality health care for astronauts through the efficient integration of medical information technologies in its system of care. Requirements should be determined by appropriate risk assessments of specified missions. The groups also encouraged NASA to collaborate with the public and the private sectors to maximize mission success through continuous quality improvement.

Generally, requirements cover two functional areas: (1) pre-flight preparation; and (2) in-flight operations. The latter are the most critical due to limited in-flight resources such as weight, volume, power, bandwidth, etc. Requirements are further categorized to focus on prevention, monitoring, diagnosis and decision support, and intervention (treatment).

Pre-Flight Requirements: Crew Selection. Crew members should be screened thoroughly for relevant physical and psychological attributes as well as specific health risks that might affect performance and endurance during space flight. Pertinent personal and family medical histories should be included in the assessment. Crew members should be selected on the basis of "wellness" status, supported and determined by reliable and precise health status indicators including diagnostic data derived from imaging, laboratory analysis (gas, fluid, and tissue) and advanced physical diagnostics technology. Medical screening must be based on state-of-the-art physical and mental diagnostic modalities to the fullest extent possible.

In-Flight Requirements: Crew Members. Especially in long-duration space flight, crews will have limited communications capabilities. Continuous "real time" or instantaneous communication with Mission Control will not be possible beyond a certain distance from or position relative to earth. As missions extend to greater distances from the earth, communication latency (lag time) will increase. Accordingly, the level of on-board medical skills and capabilities will need to evolve to increasing degrees of autonomy to support such mission profiles. For example, latency and response time, defined here as the amount of time required for a packet of information or data to be transmitted between communicating entities on a network, from the surface of Mars could take as long as 40 minutes. And, depending on the relative orbital positions of the earth and Mars, there could be a total absence of communications for periods of time lasting several hours. Therefore, (1) the crew must be able, when necessary, to function as an autonomous unit with regard to certain health and medical considerations; (2) at least two crew members should be cross-trained to be 'crew medical officers' (CMO). The CMOs should receive extensive training in space medicine and physiology and be able to utilize fully onboard systems and technologies to assess health status, and intervene when necessary. Ideally, at least one CMO should be a physician. In addition, all crew members should be trained in health maintenance and prevention, and possess basic emergency health and medical skills.

Interventions. Preventive health measures include the provision of appropriate physical and psychosocial support and/or environments. These include nutrition, an appropriate balance of work, rest, recreation, exercise, and health status monitoring, as well as appropriate interpersonal, motivational, and other psychosocial support as necessary.

Telemedicine and Medical Informatics Requirements. The medical concept of operation for long duration and expedition-class missions envisions the medical support infrastructure functioning as a seamless enterprise consisting of various nodes (spacecraft, multilateral mission control centers, and multiple terrestrial experts/consultants). Key elements of a 'Mission Extended Medical Enterprise' include user interfaces and displays, intelligence and automated smart systems, sensors, and data acquisition systems. Specifically, an adaptive, integrative medical care capability is required to address a broad range of medical issues, including emergency, acute, and even chronic conditions. The system requires a degree of sophistication adequate for diagnosis and autonomous feedback. Virtual "tools" and environments are required to support autonomous crew medical diagnoses and interventions. Information systems and smart systems tech- 
nologies must be integrated to provide enhanced synergistic medical and health care capabilities.

Approaches for Achieving Vision and Meeting Requirements. The working groups identified practical methodologies that might be employed to achieve goals enunciated in the vision statements and to meet explicit requirements. To some extent, therefore, these approaches may also be viewed as requirements. The working groups proposed a number of conceptual, practical, and technical "approaches" to address the stated vision and requirements. Specifically, it was recommended that a comprehensive needs assessment must form the basis for developing an appropriate space medicine program supported by telemedicine and medical informatics. Based on knowledge of terrestrial and space medicine, probabilities should be assigned to a range of health and medical problems or scenarios that may occur. For instance, there is evidence of potentially serious problems due to physiological changes resulting from long exposure to microgravity, including those related to loss of bone matter and muscle strength, atrophy of certain muscles, particularly those of the heart, and, a decrease in the number of oxygen-carrying cells. To the extent possible, effective technology and communication systems and connectivity essential to each scenario must be developed for those situations when astronauts are in contact with ground control and also when they are not.

Collaboration, cooperation, and integration with other organizations in the public and private sectors were deemed necessary for the most efficient and effective approach to fulfilling both the vision statements and requirements. Hence, appropriate avenues for collaboration and cooperation must be explored with other federal agencies such as the Department of Defense (DoD), National Institutes of Health (NIH), and the National Library of Medicine (NLM). In addition, NASA should continue to monitor, invite, and solicit input from industries and academic centers of excellence dealing with related medical issues and/or technology.

Technical Approaches. The major focus of recommendations regarding the vision statements and requirements, not surprisingly, were directed toward the technological aspects of space medicine. Recommendations for technology that relate to health status monitoring, assessment, diagnosis, and intervention, as well as communication systems, were included. However, the level of discourse for these approaches was necessarily generic. Furthermore, the technical recommendations for optimal performance were offered without budget considerations, even though the participants were well aware of the importance of cost.

Technical recommendations listed in Table 1 address communications issues, application and integration of sensors, effective clinical analysis and assessment tools, and appropriate intervention and treatment tools. Participants recommended that appropriate cost/risk/benefit analyses should be conducted and considered in the actual practice of contemplating and planning for specific space flight missions.

The working groups emphasized that this list of requirements was necessarily incomplete and is merely "reflective" of the general thinking on the matter. Further limitations and concerns to be considered include bandwidth issues, storage/volume of equipment and medical supplies in competition with other needs and total payload, shelf life, contemporary knowledge base, mission travel time and "remoteness," and of course communication access, quality, and response time.

Final Recommendations. The final task of the working groups was to develop final recommendations. Each group was asked to propose recommendations it deemed most critical for continuing not only the development of a strategic vision for telemedicine and medical informatics in support of space flight but also the implementation of his vision. In view of the significant overlap in recommendations, they are presented here as one set.

The issues of integration and collaboration/partnerships emerged in one form or another in the final recommendations of all groups. It was recommended that integration be both vertical and horizontal.

Integration is required within NASA between engineering and medical operations, technical systems and engineering, the hu- 
Table 1. Technical Recommendations

\section{Communication}

1. An onboard high bandwidth capability is necessary with independent elements (e.g., imaging instruments should have sufficient independent storage, processing and display capabilities).

2. There should be a central repository with high bandwidth store-and-forward capability.

3. Real time data accessibility on board.

4. On-board wireless technology sufficient to handle data management and communications.

5. Establishment of "relay" satellites to minimize interruption to communications resulting from location and position of spacecraft.

\section{Sensors}

1. Minimally invasive sensors including: (a) imaging sensors (e.g., CT, MRI, DEXA); (b) ionizing radiation sensors for spacecraft and crew members; and (c) gas and fluid content assessment sensors.

Note: Sensors should be integrated into the distributed system and, where possible, embedded in equipment and crew members.

Clinical analysis and assessment

1. Established protocols with supervision and latitude when necessary.

2. Adequate decision support.

3. Education and mentoring.

4. Robotic surgery capabilities.

5. Virtual environments with haptic feedback.

Intervention and treatment

1. Smart devices and products.

2. Simulation models.

3. Decision support and protocol supervision.

4. On-board pharmaceuticals and formulary.

5. On-board capability and expertise to make and/or assemble special purpose medical instruments and medications.

6. Robotic surgery capabilities.

7. Crew member isolation facilities as required.

8. Crew member recovery programs and facilities including recreation and psychosocial support; and should death occur.

9. Appropriate techniques and equipment necessary for disposal or storage of remains.

man-machine interface, and medical hardware and all platforms. This integration must be based on interoperability, interchangeability, and common platforms.

Collaboration and partnership development is required between field centers and operations of NASA, operational medicine and biomedical science research and development, NASA and federal departments or agencies (DOD, NIH, NLM, etc.), and private industry, academia, and other governments as partners. Partnerships should also be developed between operational medicine and biomedical science research and development, and the community of scholars working in related areas.

To maximize the contribution of telemedicine and medical informatics to space flight, a needs assessment must be conducted in several important areas. This must include a thorough review of past medical problems encountered by astronauts, "true" medical and health needs attending future space flight, and the proba- bilities of a range of illnesses and injuries during space flight.

Related to and perhaps derived from need assessment, training would be necessary for space medicine practitioners in information technology, telemedicine, and medical informatics, and their integration and application in space flight.

NASA can serve as a test bed to validate disparate medical technologies and capabilities to assist in creating an international set of standards through the use of the space station program and the International Space Station as a test bed. Also, validation and verification of hardware (including autonomous systems) and personnel (in-flight and ground-based practitioners) can be achieved through a NASA program of testing and evaluation.

Finally, the development of a telemedicine/medical informatics-based space flight medical program must be developed on the basis of robust communications systems. The 
level of communications requirements for each type of medical scenario (determined by needs assessment) must be ascertained; and, outreach is necessary to public and government secure adequate levels of funding for space flight medicine development.

\section{SUMMARY}

This Workshop was designed to assist in the ongoing development and application of telemedicine and medical informatics to support extended space flight. Participants included specialists in telemedicine and medical/health informatics (terrestrial and space) medicine from NASA, federal agencies, academic centers, and research and development institutions located in the United States and several other countries. The participants in the working groups developed vision statements, requirements, approaches, and recom- mendations pertaining to developing and implementing a strategy pertaining to telemedicine and medical informatics. Although some of the conclusions and recommendations reflect ongoing work at NASA, others provided new insight and direction that may require a reprioritization of current NASA efforts in telemedicine and medical informatics. This, however, was the goal of the Workshop. NASA is seeking other perspectives and views from leading practitioners in the fields of telemedicine and medical informatics to invigorate an essential and high-priority component of the International Space Station and future extended exploration missions. Subsequent workshops will further define and refine the general findings and recommendations achieved here. NASA's ultimate aim is to build a sound telemedicine and medical informatics operational system to provide the best medical care available for astronauts going to Mars and beyond.

\section{APPENDIX A}

List of Participants In the Workshop With Affiliation

Jake Angel

David Balch, MA

Michael Barratt, MD

William S. Barry, MD

Rashid L. Bashshur, PhD

Roger D. Billica, MD

Genie Bopp

Oscar W. Boultinghouse, MD

Timothy Broderick, MD

Douglas Butler

Jonathan B. Clark, MD

Richard L. Craft, MS

Volker R. Damann, MD

Jeffrey R. Davis, MD, MS

David L. Dawson, MD

Charles R. Doarn, MBA

Charles H. Evans, Jr., MD, PhD

Earl W. Ferguson, MD

Craig Fischer, MD

Michael W. Freckleton, MD

Christoph R. Kaufmann, MD, MPH
University of Texas Medical Branch

East Carolina University School of Medicine

NASA Johnson Space Center

NASA Kennedy Space Center

University of Michigan Health System

NASA Johnson Space Center

Wyle Laboratories

University of Texas Medical Branch

Virginia Commonwealth University (VCU)

Wyle Laboratories

NASA Johnson Space Center

Sandia National Laboratories

European Space Agency European Astronaut Center

University of Texas Medical Branch

NASA Johnson Space Center

VCU Medical Informatics and Technology Applications Consortium

National Academy of Sciences, Institute of Medicine

Southern Sierra Medical Clinic

NASA Johnson Space Center

Wilford Hall Medical Center

Uniformed Services University of the Health Sciences 
Andrew W. Kirkpatrick, MD Corinna (Cori) E. Lathan, PhD James S. Logan, MD, MS

Vernon McDonald, PhD

Norman E. McSwain, Jr., MD

Ronald C. Merrell, MD

Vincent L. Pisacane, PhD

Sam L. Pool, MD

Muriel Ross, PhD

Jay H. Sanders, MD

Consortium V. Sarohia, PhD

G. Rufus Sessions, PhD

Kaz Shimada, MD

Scott C. Simmons, MS

Jack W. Smith, M.D., PhD

Don Stilwell

Steven F. Viegas, MD

CW Vowell

David R. Williams, MD
Vancouver Hospital \& Health Sciences Centre

The Catholic University of America

JSC / Kelsey-Seybold

Wyle Life Sciences

Tulane University School of Medicine

Virginia Commonwealth University-Medical College of Virginia

Johns Hopkins University Applied Physics Laboratory

NASA Johnson Space Center

University of New Mexico Health Sciences Center

VCU Medical Informatics and Technology Applications

NASA Jet Propulsion Laboratory

Telemedicine and Advanced Technology Research Center (TATRC)

JAMSS

Wyle Life Sciences

University of Texas-Houston

NASA Johnson Space Center

University of Texas Medical Branch

JAMSS America

NASA Johnson Space Center

\section{REFERENCES}

1. Doarn CR, Nicogossian AE. NASA's Integrated Strategic Program Plan for Telemedicine: Advanced Information and Telecommunications for Health Care. March 1997.

2. Doarn CR, Nicogossian AE, Merrell RC. Application of telemedicine in the United States space program. Telemed I 1998;4:19-30.

3. NASA Space technology applied to rural Papago advanced health care. NASA Publication JSC-09161. Washington, DC; NASA Headquarters; 1974.

4. Bashshur RL, Lovett J. An Assessment of telemedicine: results of the initial experience. Aviation, Space Environ Med 1977;January:65-70.

5. Bashshur, RL. Technology Serves the People US Government Printing Office, 1981.

6. Houtchens BA, Clemmer TP, Holloway HC, Kiselev AA, Logan JS, Merrell RC, Nicogossian AE, Nicogossian HA, Rayman RB, Sarkisian AE, Siegel JH. Telemedicine and international disaster response: medical consultation to Armenia and Russia via a telemedicine Spacebridge. Prehosp Disast Med J 1993;8:57-66.

7. Angood PB, Doarn CR, Holaday L, Nicogossian AE, Merrell RC. The spacebridge to Russia project: Internet-based telemedicine. Telemed J 1999;4:305-311.

8. Simmons SC. A technology with space flight and terrestrial health care applications. Presented at the $25^{\text {th }}$ International Conference on Environmental Systems. San Diego, CA. 1995.

Address reprint requests to: Charles R. Doarn, M.B.A. Executive Director Medical Informatics and Technology Applications Consortium NASA Commercial Space Center Virginia Commonwealth University

P.O. Box 980480

Richmond, VA 23298

E-mail: crdoarn@hsc.vcu.edu 